This item was submitted to Loughborough's Research Repository by the author.

Items in Figshare are protected by copyright, with all rights reserved, unless otherwise indicated.

\title{
Wave-like aquatic propulsion of mono-hull marine vessels
}

PLEASE CITE THE PUBLISHED VERSION

http://dx.doi.org/10.1016/j.oceaneng.2009.12.008

PUBLISHER

(c) Elsevier

VERSION

AM (Accepted Manuscript)

LICENCE

CC BY-NC-ND 4.0

REPOSITORY RECORD

Krylov, Victor V., and E. Porteous. 2012. "Wave-like Aquatic Propulsion of Mono-hull Marine Vessels". figshare. https://hdl.handle.net/2134/10791. 
This item was submitted to Loughborough's Institutional Repository (https://dspace.lboro.ac.uk/) by the author and is made available under the following Creative Commons Licence conditions.

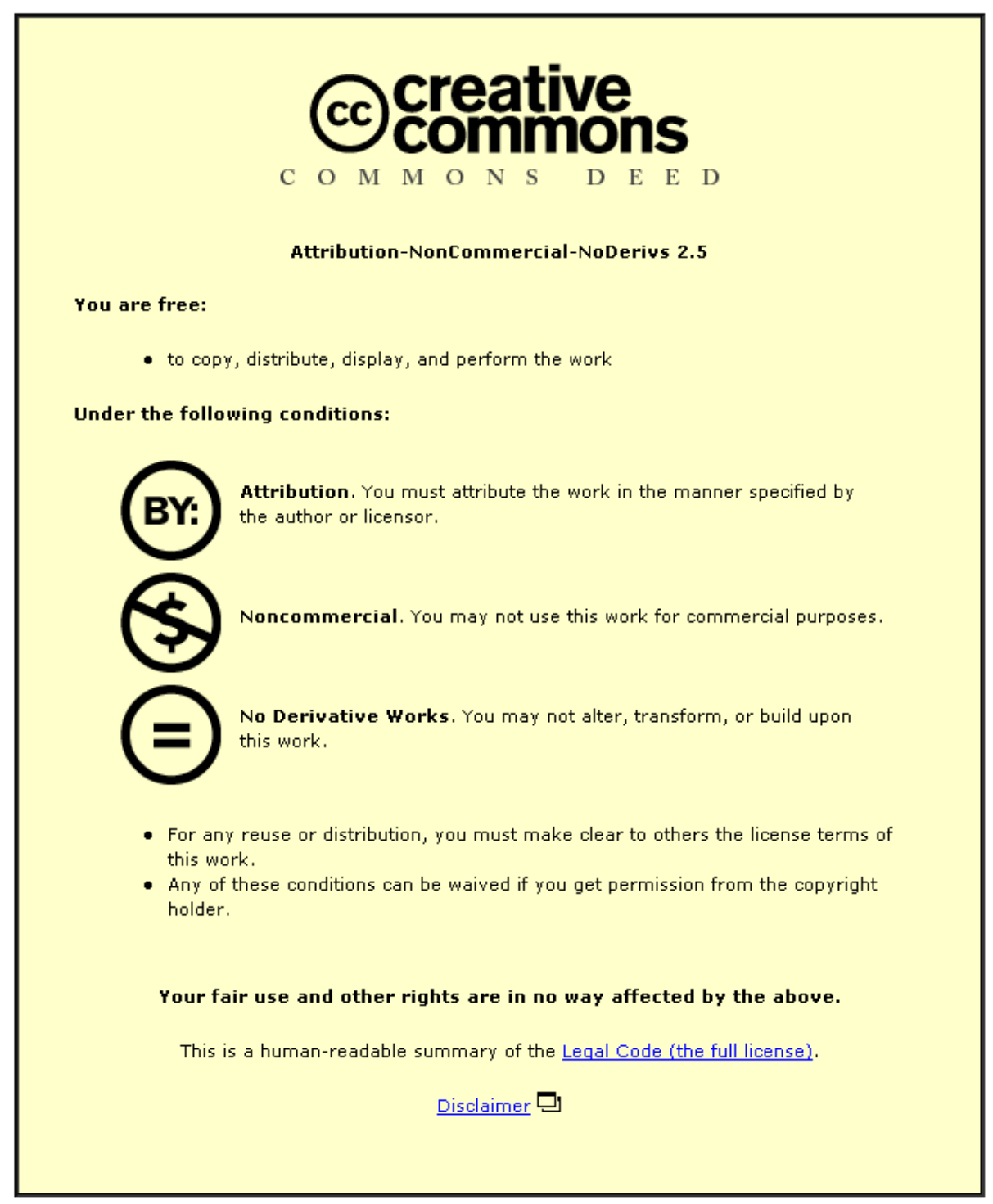

For the full text of this licence, please go to: http://creativecommons.org/licenses/by-nc-nd/2.5/ 


\title{
Wave-like aquatic propulsion of mono-hull
}

\section{marine vessels}

\author{
V.V. Krylov* and E. Porteous \\ Department of Aeronautical and Automotive Engineering, \\ Loughborough University, \\ Loughborough, Leicestershire LE11 3TU, UK
}

\begin{abstract}
The present paper describes the results of the experimental investigation of a smallscale mono-hull model boat propelled by a localised flexural wave propagating along the plate of finite width forming the boat's keel. Forward propulsion of the boat was achieved through flexural wave propagation in the opposite direction, which is similar to the aquatic propulsion used in nature by stingrays. The model boat under
\end{abstract}

\footnotetext{
${ }^{*}$ Corresponding author. Tel.: +44 1509 227216;

E-mail address: V.V.Krylov@lboro.ac.uk
} 
consideration underwent a series of tests both in a Perspex water tank and in the experimental pool. In particular, the forward velocity of the boat has been measured for different frequencies and amplitudes of the flexural wave. The highest velocity achieved was $32 \mathrm{~cm} / \mathrm{s}$. The thrust and propulsive efficiency have been measured as well. The obtained value of the propulsive efficiency in the optimum regime was $51 \%$. This indicates that efficiency of this type of aquatic propulsion is comparable to that of dolphins and sharks (around 75\%) and to that of a traditional propeller (around 70\%). In contrast to a propeller though, the wave-like aquatic propulsion has the following advantages: it does not generate underwater noise and it is safe for people and marine animals.

Key words: Wave-like aquatic propulsion, localised flexural waves, generated thrust, propulsion efficiency.

\section{Introduction}

It is well known that the most common method of aquatic propulsion used in existing marine vessels is a screw propeller. It has a simple design and is capable of propelling marine craft at high speeds. However, the conventional propeller has a number of disadvantages. In particular, these are cavitation and generation of the associated underwater noise. The 
collapsing cavitation bubbles also cause a gradual destruction of propeller blades, which limits their service life. To overcome the above problems, scientists and engineers were trying for years to create propulsive systems that could be alternatives to a propeller. Some of them were looking for inspiration in nature, trying to simulate fish swimming using elastic wave propagation in different submerged structures.

In particular, Botman (1965) investigated the feasibility of using a mechanically excited undulating plate to propel a model catamaran. He has demonstrated experimentally that this type of propulsion is feasible and it has a number of advantages over a propeller, such as the absence of shaft-sealing problem, low underwater noise (due to the absence of cavitation), safe environment for swimmers, small idling drag and good thrust control. Paidoussis (1976) used a similar model catamaran with a submerged undulating plate. However, the propagating plate flexural waves in his experiments were excited not mechanically, but as a result of hydroelastic instability (flutter) induced by a fluid flow of sufficiently high velocity. Speaking of the advantages of this method of wave-like propulsion, Paidoussis (1976) also mentions the possibility of avoiding sealing of rotating propeller shafts. In addition, he indicates the ability of the method to propel vessels in muddy and weed-infested environments. Note that in both investigations mentioned above the authors tried to emulate the so-called 'anguilliform' fish swimming mode, which is a subcategory of the more general type of body and/or caudal fin locomotion (BCF) (Sfakiotakis et al., 1999; Paidoussis, 2004).

In more recent works, the anguilliform and other types of BCF locomotion have been subjected to a great deal of investigations and engineering imitations, using undulating plates and simple oscillators (Triantafyllou and Triantafyllou, 1995; Triantafyllou et al., 2000; Yamamoto et al., 1995; Sfakiotakis et al., 1999; Wolfgang et al., 1999; Guo et al., 2003; Guglielmini et al., 2003; Schouveiler et al., 2005). One should note, however, that practical applications of anguilliform and other types of BCF propulsion are limited to unmanned 
autonomous underwater vehicles (AUV), which are used for research and surveillance operations. For manned vessels the anguilliform and BCF propulsion in its traditional form is unsuitable, as the main body of the vessel would be subject to intensive vibrations in reaction to the propulsion, which would make onboard conditions unsustainable. To overcome this disadvantage, a symmetric configuration with dual-foil counter-phase propulsors can be used (see Jones et al., 1999; Liu, 2005; Terada et al., 2006). In such configuration side forces cancel each other, with only a production of thrust force from the entire propulsion system. However, such dual systems are rather complex and not easy to actuate. Apart from this, they should be perfectly balanced to provide the cancellation of side forces. For the above reasons, emulating the so-called median and/or paired fin (MPF) locomotion seems to be more suitable for manned vessels. One of the subcategories of this locomotion, called 'rajiform', is used in nature by stingrays and skates (Sfakiotakis et al., 1999).

The idea of wave-like aquatic propulsion of manned marine vessels to be dealt with in this paper has been first published by Krylov (1994). This idea is based on employing the rajiform type locomotion and is implemented using the unique type of localised flexural elastic waves propagating along edges of wedge-like structures immersed in water (wedge elastic waves). Such wedge-like structures supporting localised elastic waves are to be attached to a body of a small ship or a submarine as keels or wings that are to be used for aquatic propulsion. The above-mentioned wedge elastic waves propagating in contact with water have been first predicted and investigated theoretically by Krylov $(1994,1998)$. The principle of using localised elastic waves as a source of aquatic propulsion is similar to that used in nature by stingrays, which utilise wave-like motions of their large horizontal pectoral fins (wings) for moving forward. It is vitally important for the application of localised elastic waves for propulsion of manned marine vessels that, in spite of vibration of the fins, the main 
body of the craft remains undisturbed because the energy of localised waves is concentrated near the wings’ tips (Krylov, 1994).

Thus, the main advantage of this kind of wave-like propulsion of marine craft over the existing methods using undulating plates, e.g. the ones described by Botman (1965) and Paidoussis (1976), is that the main body of the craft is isolated from fin vibrations. This permits this method to be used for propulsion of manned vessels. The advantages of this method, in comparison with traditional methods of propulsion such as jets and propellers, are largely the same as those mentioned by Botman (1965) and Paidoussis (1976). Namely, the absence of propeller shaft-sealing problem, low underwater acoustic noise (due to the absence of cavitation), safe environment for swimmers, small idling drag, good thrust control, and the ability to propel vessels in muddy and weed-infested environments.

The main envisaged applications of the proposed type of wave-like propulsion are small and medium manned research submarines and autonomous underwater vehicles (AUV). Other possible applications can be for small and medium surface marine vessels, in particular for sailing boats. As it is well known, sailing boats are stranded in times of low wind, which is usually overcome with outboard motors. The replacement of the outboard motor by a flexible keel supporting wave-like propulsion, in place of the usual centre/dagger board, would have significant advantages. In particular, the propulsive keel would not interfere with the hydrodynamic characteristics of the hull.

The first practical implementation and experimental testing of this type of aquatic propulsion have been carried out recently using a small model catamaran employing localised flexural waves propagating in a vertical rubber plate (Krylov et al., 2007). The test results have shown that the catamaran was propelled quite efficiently and could achieve the speed of $36 \mathrm{~cm} / \mathrm{s}$ (or about one body length per second), thus demonstrating that the idea of wave-like aquatic propulsion of manned craft is viable. 
The present paper describes the design and experimental testing of a small-scale monohull model boat (see Fig. 1) propelled by a localised flexural wave propagating along a rubber plate of finite width forming the boat's keel. Tests include measurements of boat speed as well as measurements of thrust and propulsion efficiency. The model boat under consideration is fully autonomous and robotically controlled. The results of the tests performed on the model boat confirm that wave-like propulsion using localised flexural waves is an attractive method of propulsion for mono-hull aquatic craft. It has been found that increasing both frequency and amplitude of the waves results in an increase in thrust. The efficiency of the above wave-like propulsion system was found to be $51 \%$. This is comparable to the $70 \%$ efficiency typical for propellers, and to the 75\% efficiency typical for dolphins and sharks. However, the efficiency should not be considered as the most important feature of wave-like aquatic propulsion. The other benefits, such as the absence of cavitation and of the associated underwater noise, as well as environmentally friendly operation, make this type of aquatic propulsion very interesting for many practical applications.

\section{Localised flexural waves used for aquatic propulsion}

As was discussed earlier (Krylov, 1994; Krylov et al., 2007), ideally a propulsive plate structure should have a wedge-like quadratic profile to provide full isolation of the flexural wave energy from the craft's body. This however was not implemented in this investigation due to the time and cost constraints. Like in the earlier experimental work (Krylov et al., 2007), a wedge was therefore replaced by a plate of constant thickness, with one of its horizontal edges being clamped and another one remaining free. 
One should keep in mind however that, in contrast to quadratic wedges, such 'clampedfree' plates do transmit vibrations to the main body of a vessel through the area of clamping. Therefore, although quite suitable for autonomous under-water vessels (AUV), the aforementioned 'clamped-free' rubber plates can not be recommended for applications to real manned marine vessels. For the purpose of experimental investigations on a model vessel described in this paper they, however, are perfectly acceptable. Note that all of the abovementioned localised flexural waves in contact with water are waves propagating in the subsonic regime of wave propagation (in respect of underwater sound). As it is well known, such waves ideally do not generate sound in the surrounding water. Therefore, the associated aquatic propulsion is virtually quiet, which is an attractive feature for both man-inhabited vessels and AUV.

Theoretical calculations of frequency-dependent phase velocities (dispersion curves) of the lowest-order guided flexural mode in two immersed 'clamped-free' rubber strips have been carried out recently using the geometrical acoustics approach (Krylov et al., 2007). Both strips were of the same width $H=150 \mathrm{~mm}$, but had different values of thickness: $h=1 \mathrm{~mm}$ and $h$ $=3 \mathrm{~mm}$. According to the above calculations, the dispersion curve for a strip of $1 \mathrm{~mm}$ thickness is almost indistinguishable from the dispersion curve for an infinite plate of the same thickness. However, for a thicker strip $(h=3 \mathrm{~mm})$ the dispersion curve differs slightly from the corresponding infinite plate curve and has a minimum at frequency of $0.2 \mathrm{~Hz}$ (Krylov et al., 2007). Note that in the case under consideration the calculated phase velocities of flexural waves in immersed 'clamped-free' rubber strips are very low. For example, for a strip of $1 \mathrm{~mm}$ thickness they vary from about $2 \mathrm{~cm} / \mathrm{s}$ at frequency of $0.2 \mathrm{~Hz}$ to $24 \mathrm{~cm} / \mathrm{s}$ at frequency of $8 \mathrm{~Hz}$ (these are much lower than wave velocities in the same strips and plates in vacuum). For a strip of $3 \mathrm{~mm}$ thickness the velocities vary from about $9 \mathrm{~cm} / \mathrm{s}$ at frequency of $0.2 \mathrm{~Hz}$ (corresponding to the velocity minimum) to $49 \mathrm{~cm} / \mathrm{s}$ at frequency of $8 \mathrm{~Hz}$. Such low wave 
velocities have been specifically chosen to provide several wavelengths of flexural waves on the length of the propulsive fin, which was required to emulate the 'rajiform' wave-like motion associated with stingrays.

\section{Design and construction of the model boat}

The first stage in the design and construction of the considered model boat, that will be also referred to as “Biomimetic Robotically-operated Aquatic VEhicle” (BRAVE), was to define the propulsive plate excitation method. The chosen design implements a leading edge excitation mechanism. Excitation of the leading edge in this manner causes localised wave propagation throughout the length of the propulsive plate towards the trailing edge.

As was described in the previous section, the propulsive plate material and thickness are the primary factors that determine the speed of flexural wave propagation in contact with water, and as such they are major factors which determines the maximum boat speed and efficiency. The choice of material in the present work was rubber, with the mass density $\rho=$ $1100 \mathrm{~kg} / \mathrm{m}^{3}$, shear modulus $\mu=0.001 \cdot 10^{9} \mathrm{~N} / \mathrm{m}^{2}$ and Poisson's ratio $\sigma=0.49$. The dimensions of a rectangular rubber plate were $250 \mathrm{~mm}$ in horizontal direction (chord) and 110 $\mathrm{mm}$ in vertical direction (span). The values of plate thickness were $1 \mathrm{~mm}, 1.5 \mathrm{~mm}$ and $2 \mathrm{~mm}$.

The hull of the model boat under consideration, the $\boldsymbol{B R} \boldsymbol{A V E}$, utilised an existing plastic construction developed for a radio-controlled hobby application. Utilisation of this hull provided a number of advantages. In particular, it helped to minimise construction costs and to ensure the craft's stability. The concept drawings of the propulsive plate with the exciter bar and its view under water are shown in Figs. 2 and 3 respectively. 
The propulsive rubber plate was friction fitted into the aluminium chassis slot. Note that the propulsion system was designed to ensure that, when installed, the water level lies below any through openings such as the plate slot and the exciter bar slot. This would prevent water spilling over into the hull. The exciter bar, which was driven by a servo motor, has been designed to allow maximum angle of $30^{\circ}$ to be achieved either side of the centre line (see Fig. 2). With the exciter bar length used this gave a maximum amplitude of $33 \mathrm{~mm}$.

Following construction of the $\boldsymbol{B R A V E}$, it was necessary to validate and to optimise the propulsion system. Both the experimental pool and a Perspex test tank were used for the experiments. The following variables were investigated to ascertain the effect on propulsive effectiveness: Propulsive plate thickness, Length/width of propulsive plate, Leading edge constraints, Trailing edge constraints. Figure 4 shows the underwater view pictures taken in a Perspex tank and illustrating flexural wave propagation in the propulsive plate of $1 \mathrm{~mm}$ thickness at different time instants over the full period of $333 \mathrm{~ms}$ corresponding to the operating frequency of $3 \mathrm{~Hz}$.

\section{$4 \quad$ Thrust and drag measurements}

Flexural-wave-generated thrust of the $\boldsymbol{B R A V E}$ was measured both in static position (the so-called 'bollard pull thrust') - using a spring gauge attached to the stern (see Figs. 5 and 6), and in motion ('self-propulsion thrust') - via calculation using measured steady state velocities of the craft and measured drag as a function of the craft velocity. Note that, as it can be seen from Fig. 6 (see also Fig. 1), the model boat vibrates and makes waves in lateral direction, as evidenced by the waves emanating from the boat. Ideally these lateral vibrations 
should not be present. The two main souses of lateral vibration of the boat hull are a nonsymmetric motion of the servomotor driving the mechanical arm and unbalanced hydrodynamic forces acting on the propulsive plate as a result of flexural wave propagation. These unbalanced hydrodynamic forces could be reduced by increasing the number of flexural wavelengths over the plate length. And the effect of a servomotor could be mitigated by making the actuating system more symmetric. The results of the thrust measurements will be discussed in the next section.

To measure drag, the craft was towed at constant velocity, and the tension in the tow cable was measured using the spring gauge (Fig. 7). This process was repeated for a number of different speeds. The results of the drag measurements at different speeds are shown in Fig.

8. As expected, the results can be approximated by a parabolic curve (see e.g. Batchelor, 1994), the value of the coefficient being equal to 0.0036 .

Note that, strictly speaking, the drag of a surface ship does not increase monotonically due to the water-wave resistance, which is dominated by the Froude number. This mechanism in particular cold be responsible for the observed significant scattering from the fitted curve for the velocity range $10-30 \mathrm{~cm} / \mathrm{s}$.

\section{$5 \quad$ Swimming speed and other important parameters}

\subsection{Steady state velocity of swimming}

Steady state velocity of swimming was measured by allowing the $\boldsymbol{B R} \boldsymbol{A V E}$ to accelerate to a steady state velocity. A stopwatch was used to measure the time taken to traverse a $3 \mathrm{~m}$ 
course allowing an average speed for the boat to be calculated. Figure 9 shows the measured craft velocity. As one can see from Fig. 9, as both the frequency and amplitude increase, the velocity increases as well. The decrease in velocity at around $2.4-2.8 \mathrm{~Hz}$ may be due to the plate being excited near its natural frequency. One could expect that in this condition, a standing wave is created in the plate, which is displacing water at 90 degrees to the plate, rather than the desired propagating wave (see also Krylov et al., 2007). However, as the above-mentioned plate flexural wave is of large amplitude and hence highly nonlinear, this interpretation of the observed minima as effects of plate natural frequencies should be taken with some caution.

\subsection{Self-propulsion and bollard pull thrusts}

The self-propulsion thrust is directly related to the craft steady state velocity described above. Namely, for this condition thrust is equal to the drag. Therefore, the drag curve shown in Fig. 8 was used to convert the measured steady state velocity of swimming (see Fig. 9) to the thrust force being produced by the propulsive plate for that condition. The results for the thrust determined in this way are shown in Fig. 10. Note that the obtained thrust values behave very similarly to the measured steady state velocities shown in Fig. 9. In particular, the thrust force generally increases as frequency increases. It should be remembered though that a higher thrust force does not necessarily imply a higher efficiency.

An obvious source of uncertainty in measured self-propulsion thrust values can be associated with the fact that drag measurements at the tow test (see Fig. 7) were performed with the propulsion system turned off. However, when the propulsion system was actuated for the craft speed measurements, the plate was oscillating, which could result in a somewhat 
different drag force. The estimated uncertainty for the resulting self-propulsion thrust values is about $10 \%$.

The values of the measured bollard pull thrust produced at static condition (see Figs. 5 and 6) for the $28 \mathrm{~mm}$ amplitude setting are shown in Fig. 11. For comparison, the results of the above-mentioned self-propulsion thrust measurements for the same wave amplitude are shown in Fig. 11 as well. It can be seen that the experimental curves behave quite differently, which is not surprising - these are different thrusts that are defined in different conditions.

\subsection{Strouhal number and other non-dimensional parameters}

Strouhal number, St, is a non-dimensional parameter that is frequently used in hydrodynamics. In particular, it is often employed to characterise the propulsion efficiency. For example, while dolphins, sharks and bony fish move at their preferred speed, the ratio of their tail frequency $f$ and amplitude $W_{0}$ to the swimming speed $U$, which constitutes the Strouhal Number, St $=f W_{0} / U$, falls between 0.2 and 0.4 (see Taylor et al., 2003).

Strouhal number in the present work was calculated from the steady state boat velocity with the corresponding frequency and amplitude for that condition. The results show that St for the $\boldsymbol{B R} \boldsymbol{A} \boldsymbol{V E}$ is almost independent of frequency across a wide frequency range, where it takes values roughly between 0.4 and 1 , depending on wave amplitudes. The configuration that provides the lowest $\mathrm{St}=0.402$, which is closest to the above-mentioned 'natural' maximum efficiency range: $\mathrm{St}=0.2-0.4$, is the $4.4 \mathrm{~Hz}$ frequency and the $21 \mathrm{~mm}$ amplitude of the flexural wave. 
It is useful also to give the diapasons of changes for the other two important nondimensional hydrodynamic parameters for the problem under consideration. These are Reynolds number and Froude number.

Reynolds number, Re, is defined by the expression $\operatorname{Re}=U \cdot L / v$, where $U$ is the boat swimming speed, $L$ is the length of the boat at the water line level, and $v$ is the kinematic viscosity of a fluid. The value of $v$ for fresh water is $0.011 \mathrm{~cm}^{2} / \mathrm{s}$ (see e.g. Batchelor, 1994), and the value of $L$ for the model boat under consideration is $46 \mathrm{~cm}$. Therefore, for swimming speeds of the model boat varying from $1 \mathrm{~cm} / \mathrm{s}$ to $32 \mathrm{~cm} / \mathrm{s}$ (see Fig. 9), the values of Re vary from $4.182 \cdot 10^{3}$ to $1.338 \cdot 10^{5}$ respectively.

Froude number, Fr, is defined by the formula $\operatorname{Fr}=U /(g \cdot L)^{1 / 2}$, where $U$ is the boat swimming speed, $L$ is the length of the boat at the water line level, and $g$ is gravity acceleration $\left(g=981 \mathrm{~cm} / \mathrm{s}^{2}\right)$. For the same range of boat swimming speeds, i.e. from $1 \mathrm{~cm} / \mathrm{s}$ to $32 \mathrm{~cm} / \mathrm{s}$, the values of Fr vary from $4.707 \cdot 10^{-3}$ to $1.506 \cdot 10^{-1}$.

\subsection{Flexural wavelength and velocity}

The wavelength of the flexural wave motion was measured in the Perspex test tank by inspecting photos taken using a high-speed camera. At $4.4 \mathrm{~Hz}$, roughly 2.8 wavelengths were present in the plate. This gave the value of the wave speed as $39 \mathrm{~cm} / \mathrm{s}$.

Comparing this wave speed to the steady state boat velocity at this condition, $23 \mathrm{~cm} / \mathrm{s}$, gives the wave speed to swimming speed ratio of $39 / 23=1.65$. This is in line with the theoretical results of Lighthill for the swimming of slender fish (Lighthill, 1960,1970,1971), 
according to which, for the most efficient regime, the wave speed to swimming speed ratio should be equal to $5 / 4$ (or 1.25 ).

\subsection{Propulsion efficiency}

The efficiency of the wave-like propulsion under consideration has been determined as the ratio of the measured values of useful work $\left(P_{\text {OUT }}\right)$ to the electrical energy supplied to the servo motor $\left(P_{I N}\right)$. It should be noted that the propulsion efficiency determined in this work does not take into account the power losses occured in the actual actuation system. It is thus a measure of the 'true' hydrodynamic efficiency of the wave-like propulsion only. In order to determine the power inputted just into propulsion, it was necessary to measure the power consumption when running in air (which is required to overcome the actuation losses) and when in water. The difference between these two values gives the power inputted into propulsion. All measurements have been taken for the configuration corresponding to St $=$ 0.402, i. e. at the $4.4 \mathrm{~Hz}$ frequency and the $21 \mathrm{~mm}$ amplitude of the flexural wave.

Thus, the electric power input to the propulsive plate in the optimal regime has been calculated as:

$$
P_{I N}=V \cdot I=5.9 \cdot 1.5 \cdot 10^{-3}=8.85 \mathrm{~mW} .
$$

Here $V=5.9 \mathrm{~V}$ is the voltage of the batteries, and $I=1.5 \mathrm{~mA}$ is the measured difference between electric currents consumed by the craft running in water and in the air.

The useful power output has been calculated as the product of the thrust force, $2 \cdot 10^{-3} \cdot 9.81$ $N$, and the steady state craft velocity, $0.23 \mathrm{~m} / \mathrm{s}$ : 


$$
\begin{aligned}
& P_{\text {OUT }}=\text { Thrust } \cdot \text { Steady state craft velocity } \\
& =\left(2 \cdot 10^{-3} \cdot 9.81\right) \cdot 0.23=4.5 \mathrm{~mW}
\end{aligned}
$$

Thus, the propulsion efficiency $\eta$ has been calculated using the equations (1) and (2) as:

$$
\eta=P_{\text {OUT }} / P_{I N}=(4.5 / 8.85) \cdot 100=51 \% .
$$

The obtained value of $51 \%$ indicates that the efficiency of this type of aquatic propulsion is high enough. Indeed, it is comparable to that of such effective swimmers as dolphins and sharks (around 75\%) and to that of a traditional screw propeller (around 70\%), although the efficiency of foils and propellers at small thrust can be as high as near $90 \%$.

\section{Conclusions}

The results from the testing performed on the model boat $\boldsymbol{B R A V E}$ have confirmed that wave-like propulsion using localised flexural waves is an attractive method of propulsion for mono-hull aquatic craft. Unlike conventional propulsion methods, such as a propeller, wavelike aquatic propulsion ideally does not generate underwater noise and is safe for people and marine animals.

It has been found that increasing both frequency and amplitude of the flexural wave results in an increase in thrust. It should be noted however that the highest propulsive thrust does not 
necessarily correlate to the highest propulsive efficiency, and an optimum frequency and amplitude of wave motion must be found.

The efficiency of the wave-like propulsion system for the $\boldsymbol{B R A V E}$ was found to be $51 \%$ when operating at the optimum Strouhal number of 0.402 . This is comparable to the $70 \%$ efficiency typical for propellers, and 75\% efficiency typical for dolphins and sharks.

It is anticipated that with further research and technological advances it would be possible to achieve and perhaps even exceed the efficiencies of conventional propulsion methods. However, the efficiency should not be considered as the most important feature of wave-like aquatic propulsion. The other benefits, such as the absence of cavitation and of the associated underwater noise, as well as environmentally friendly operation, make this type of aquatic propulsion very attractive for many practical applications. 


\section{References}

Batchelor, G.K., 1994. An introduction to fluid dynamics. Cambridge University Press, Cambridge.

Botman, M., 1965. Propulsion by undulating plates. Journal of Aircraft 2, 456-462.

Cheng, J.Y., Blikhan, R., 1994. Note on the calculation of propeller efficiency using elongated body theory. Journal of Experimental Biology 192, 169-177.

Guglielmini, L., Blondeaux, P., Vittori, G., 2003. A simple model of propulsive oscillating foils. Ocean Engineering 31, 883-899.

Guo, S., Fukuda, T., Asaka, K., 2003. A new type of fish-like underwater microrobot. IEEE/ASME Transactions on Mechatronics 8, 136-141.

Jones, K.D., Platzer, M. F., 1999. An experimental and numerical investigation of flappingwing propulsion. AIAA Paper No. 99-0995.

Krylov, V.V., 1994. Propagation of wedge acoustic waves along wedges embedded in water. Proc. IEEE Ultrasonics Symposium, Cannes, France, 793-796.

Krylov, V.V., 1998. On the velocities of localized vibration modes in immersed solid wedges. Journal of the Acoustical Society of America 103, 767-770.

Krylov, V.V., Shuvalov, A.L., 2000. Propagation of localised flexural vibrations along plate edges described by a power law, Proceedings of the Institute of Acoustics 22(2), 263270. 
Krylov, V.V.,. Pritchard, G.V., 2007 Experimental confirmation of the propulsion of marine vessels employing guided flexural waves in attached elastic fins, Journal of Fluids and Structures 23, 297-307.

Lighthill, M.J., 1960. Note on the swimming of slender fish. Journal of Fluid Mechanics 9, 305-317.

Lighthill, M.J., 1970. Aquatic animal propulsion of high hydrodynamic efficiency. Journal of Fluid Mechanics 44, 265-301.

Lighthill, M.J., 1971. Large amplitude elongated-body theory of fish locomotion. Proceedings of the Royal Society (London) B 179, 125-138.

Liu, P., 2005. Propulsive performance of a twin-rectangular-foil propulsor in a counterphase oscillation. Journal of Ship Research 49, 207-215.

Paidoussis, M.P., 1976. Hydroelastic ichthyoid propulsion. Journal of Hydronautics 10, 3032.

Paidoussis, M.P., 2004. Fluid-Structure Interactions, Vol. 2. Elsevier, Section 8.10.

Sfakiotakis, M., Lane, D.M., Davies, J.B.C., 1999. Review of fish swimming modes for aquatic locomotion. IEEE Journal of Oceanic Engineering 24, 237-252.

Schouveiler, L., Hover, F.S., Triantafyllou, M.S., 2005. Performance of flapping foil propulsion. Journal of Fluids and Structures 20, 949-959.

Taylor, G.K., Nudds, R.L., Thomas, A.L.R., 2003. Flying and swimming animals cruise at a Strouhal number tuned for high power efficiency. Nature 425, 707-711.

Terada , Y., Yamamoto, 2006. Robotic fish and its application. Proceedings of the IEEE Conference ‘ISIE 2006’, Montreal, Canada, 9-12 July 2006, p. 3062-3065.

Triantafyllou, M.S., Triantafyllou, G.S., 1995. An efficient swimming machine. Scientific American 272, 64-70. 
Triantafyllou, M.S., Triantafyllou, G.S., Yue, D.K.P., 2000. Hydrodynamics of fishlike swimming. Annual Review of Fluid Mechanics 32, 33-53.

Wolfgang, M.J., Yue, D.K.P., Triantafyllou, M.S., 1999. Visualisation of complex near-body transport processes in flexible-body propulsion. Journal of Flow Visualisation 2, 143151.

Yamamoto, I., Terada, Y., Nagamatu, T., Imaizumi, Y., 1995. Propulsion system with flexible/rigid oscillating fin. IEEE Journal of Oceanic Engineering 20, 23-30. 


\section{List of Figures}

Fig. 1 View of the model boat swimming in the experimental pool.

Fig. 2 Concept drawings of the propulsive plate.

Fig. 3 Underwater view of the hull and the assembled propulsive plate.

Fig 4 Wave propagation in the propulsive plate at $3 \mathrm{~Hz}$ and $20 \mathrm{~mm}$ amplitude.

Fig. 5 Spring gauge attachment

Fig. 6 Bollard pull thrust test in progress

Fig. 7 Drag measurement test

Fig. 8 Craft's drag as a function of its velocity

Fig. 9 Steady state craft velocity as a function of frequency and amplitude 
Fig. 10 Variation of self-propulsion thrust with frequency and amplitude

Fig. 11 Bollard pull and self-propulsion thrusts at $28 \mathrm{~mm}$ amplitude 


\section{Figures}

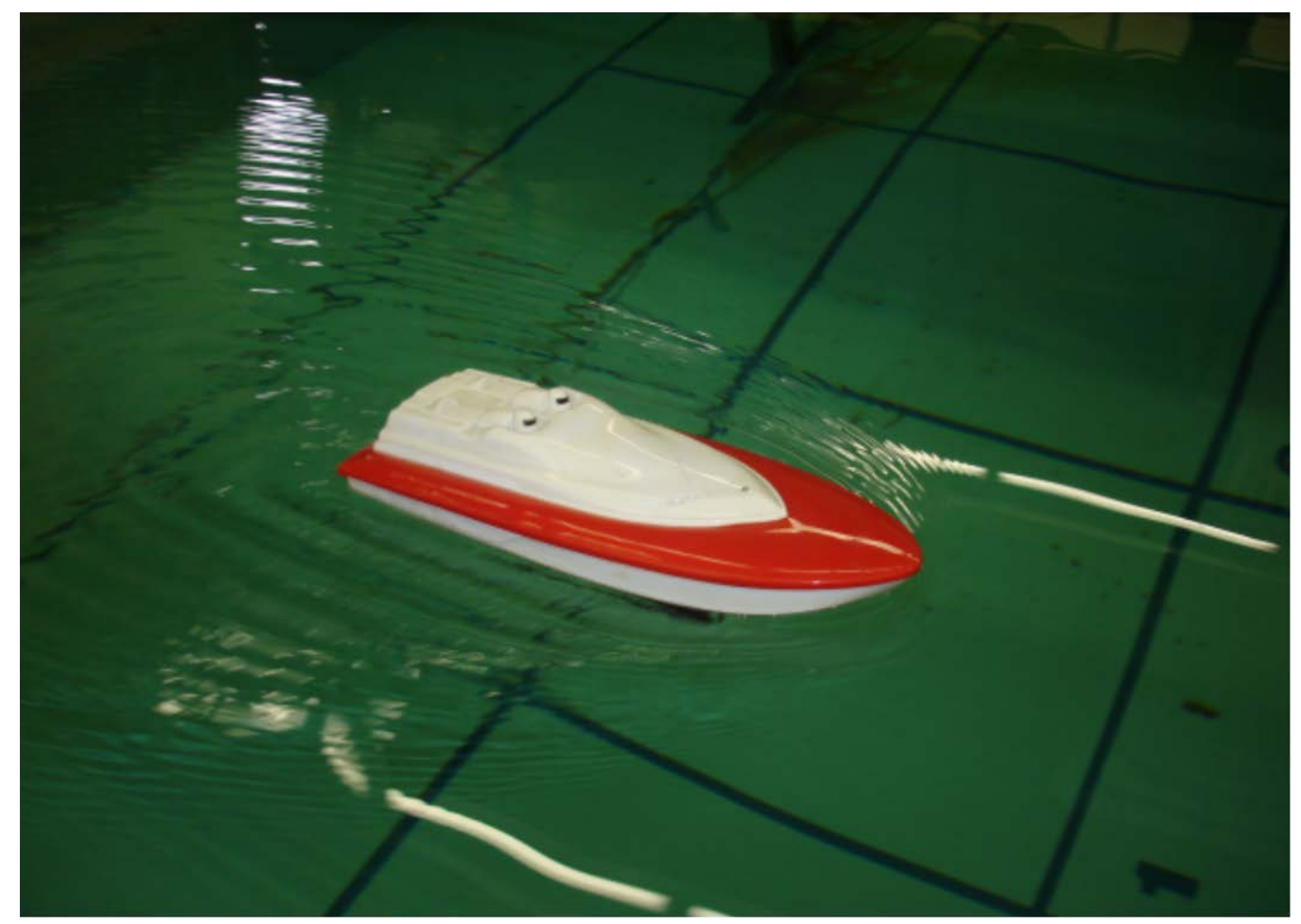

Fig. 1 View of the model boat swimming in the experimental pool. 


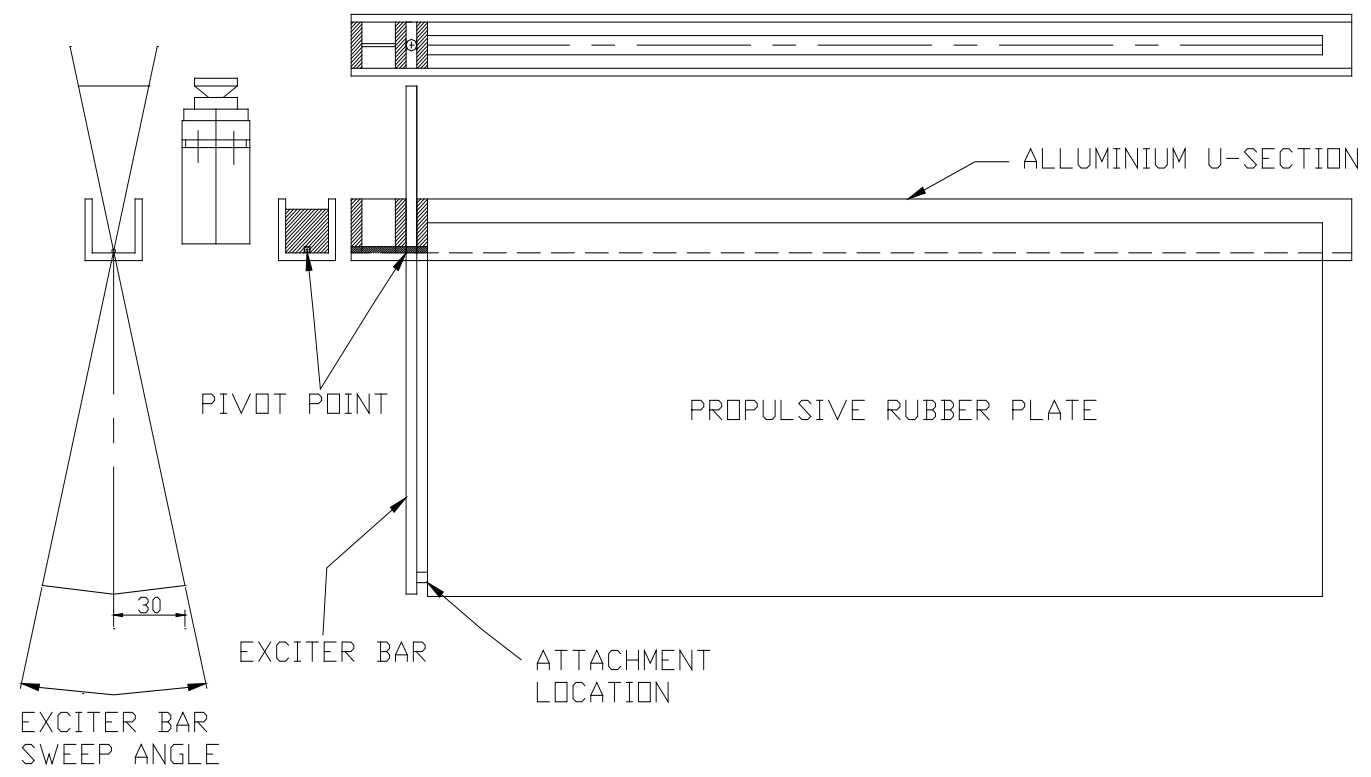

Fig. 2 Concept drawings of the propulsive plate. 


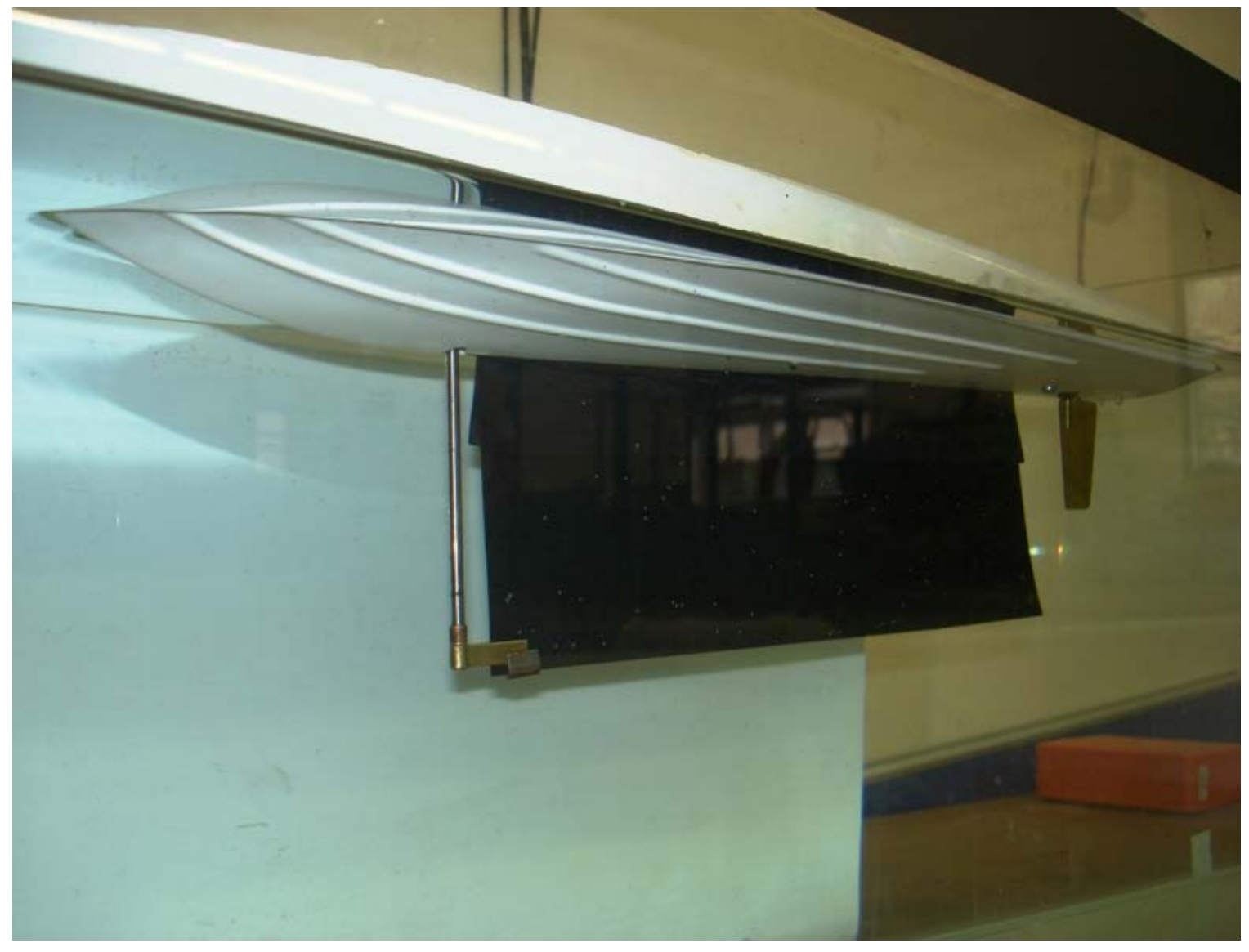

Fig. 3 Underwater view of the hull and the assembled propulsive plate. 


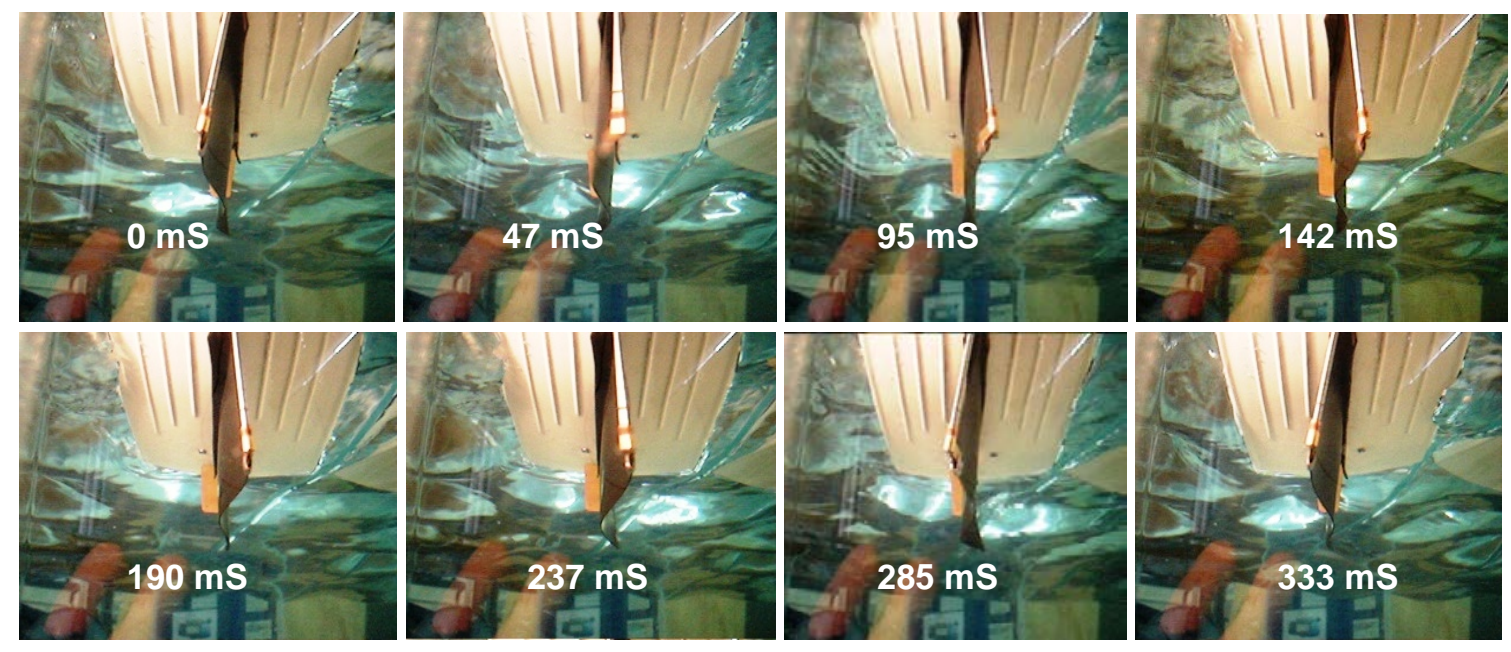

Fig. 4 Wave propagation in the propulsive plate at $3 \mathrm{~Hz}$ and $20 \mathrm{~mm}$ amplitude. 


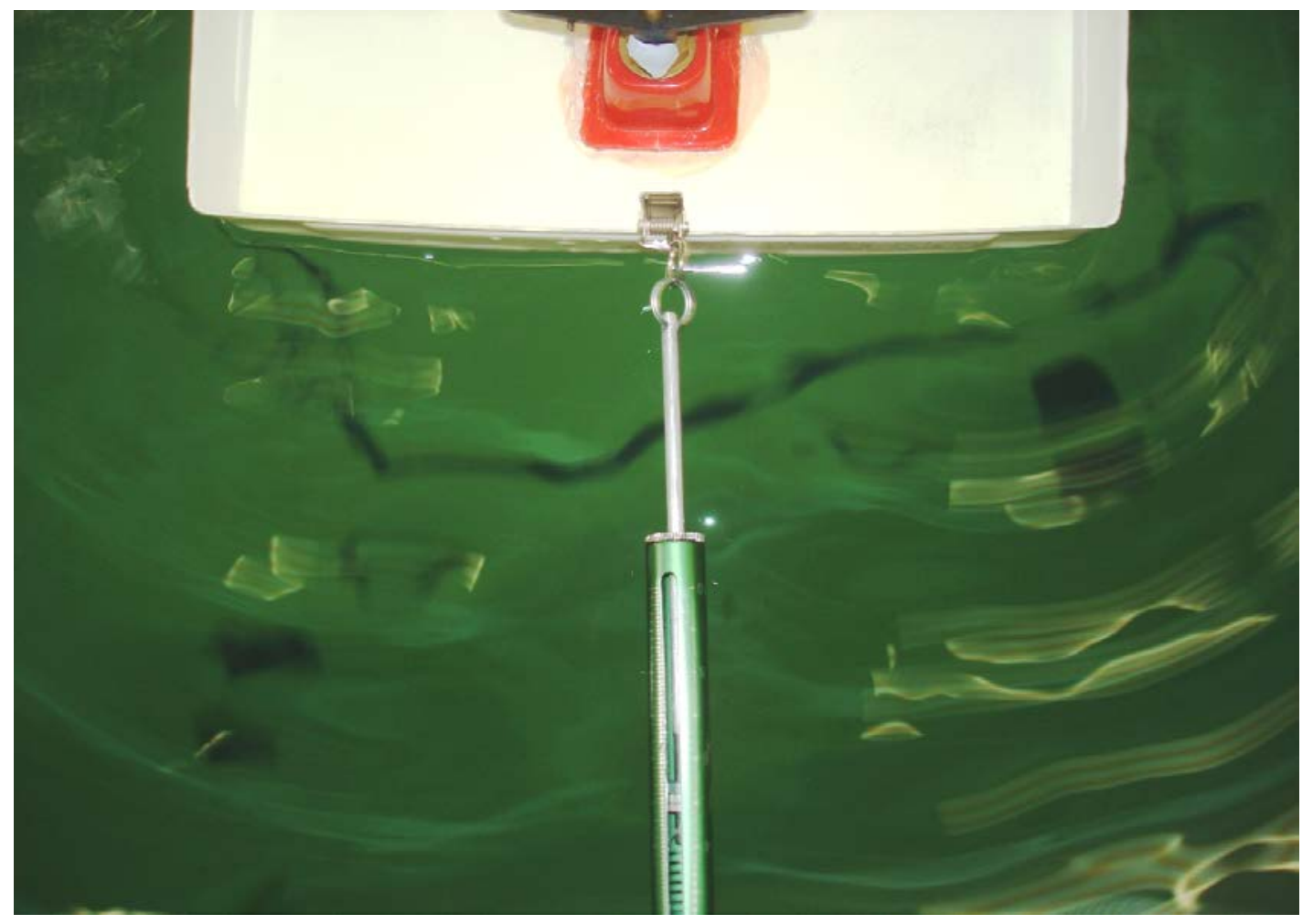

Fig. 5 Spring gauge attachment 


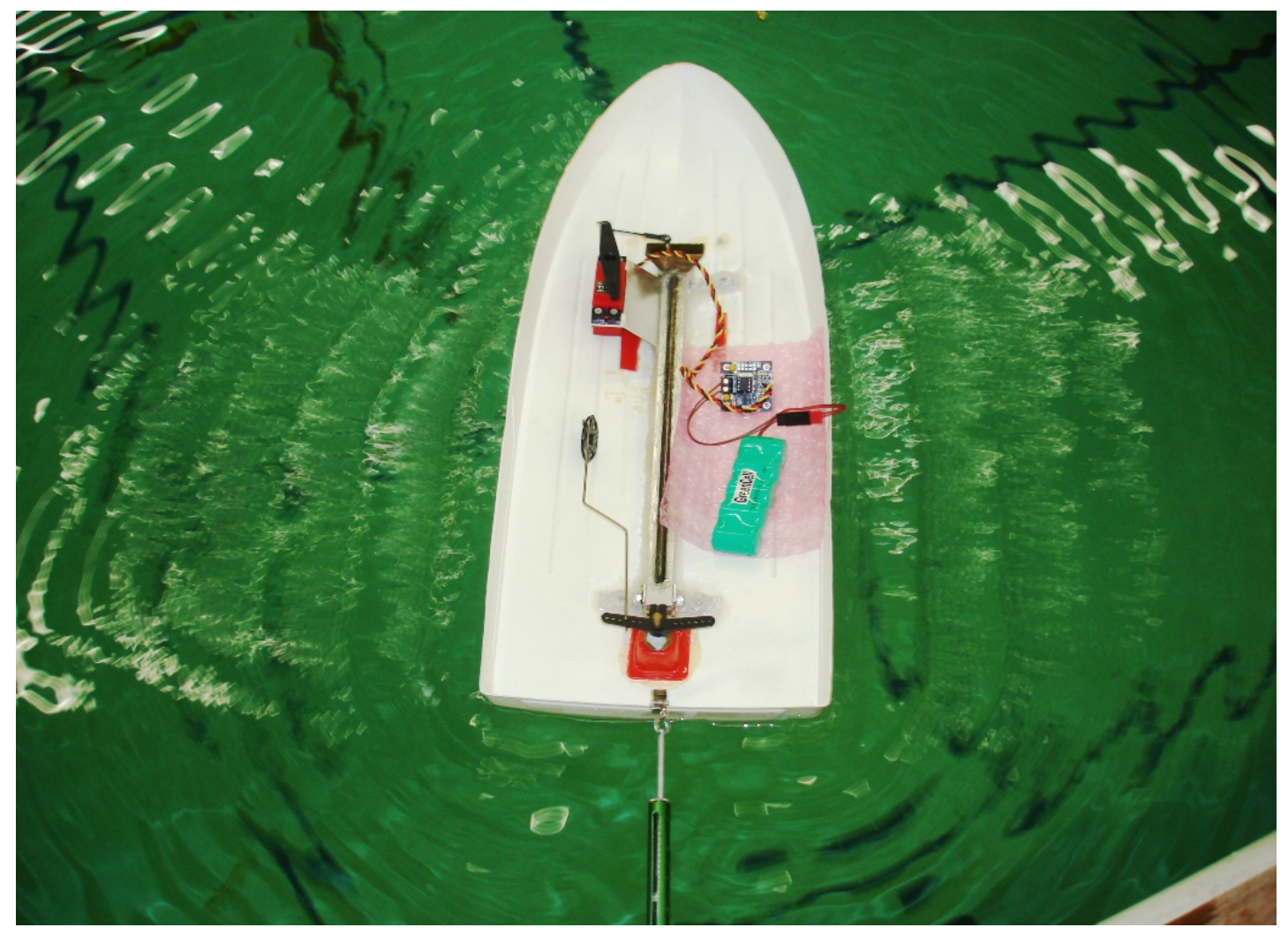

Fig. 6 Bollard pull thrust test in progress 


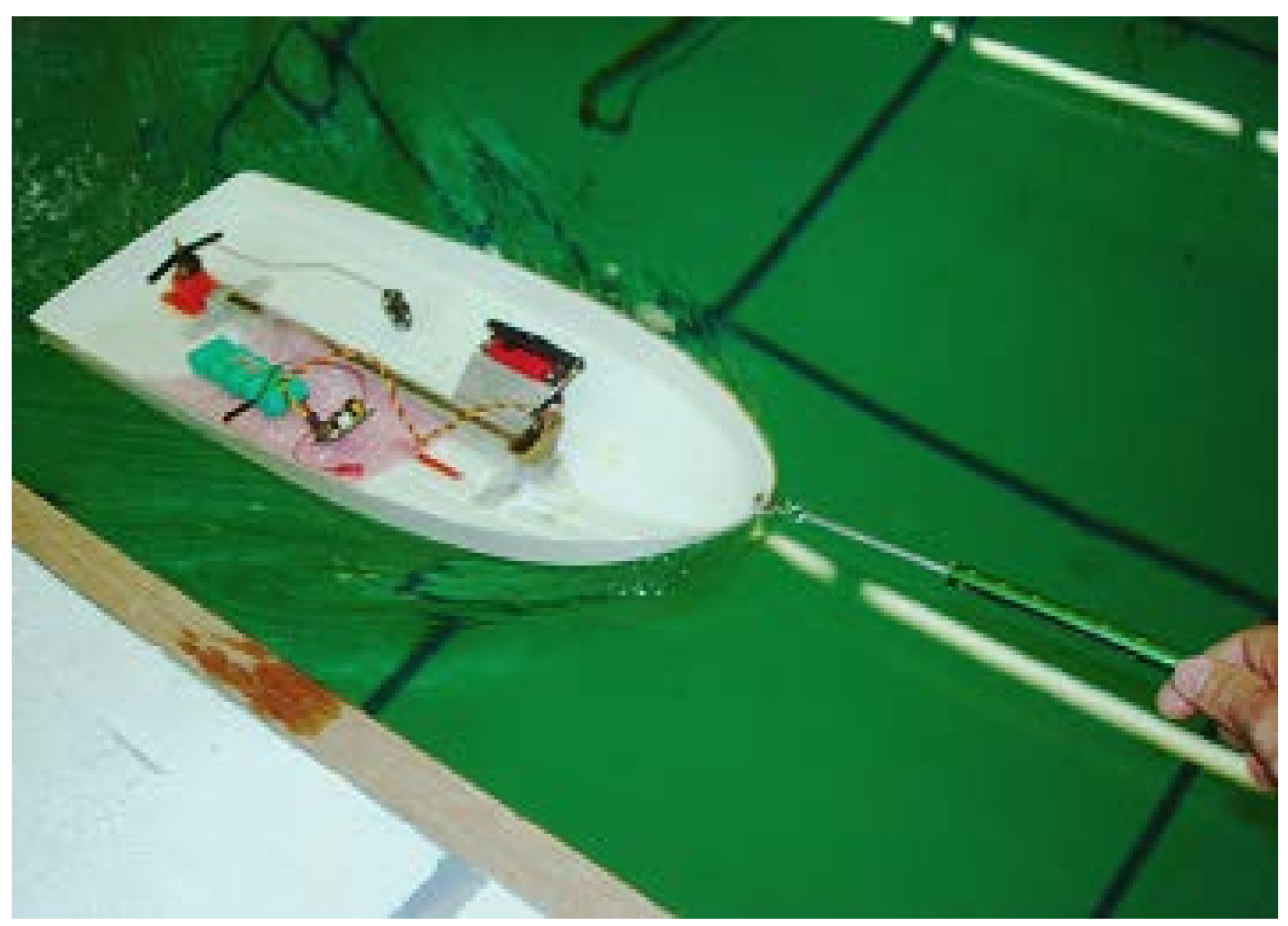

Fig. 7 Drag measurement test 


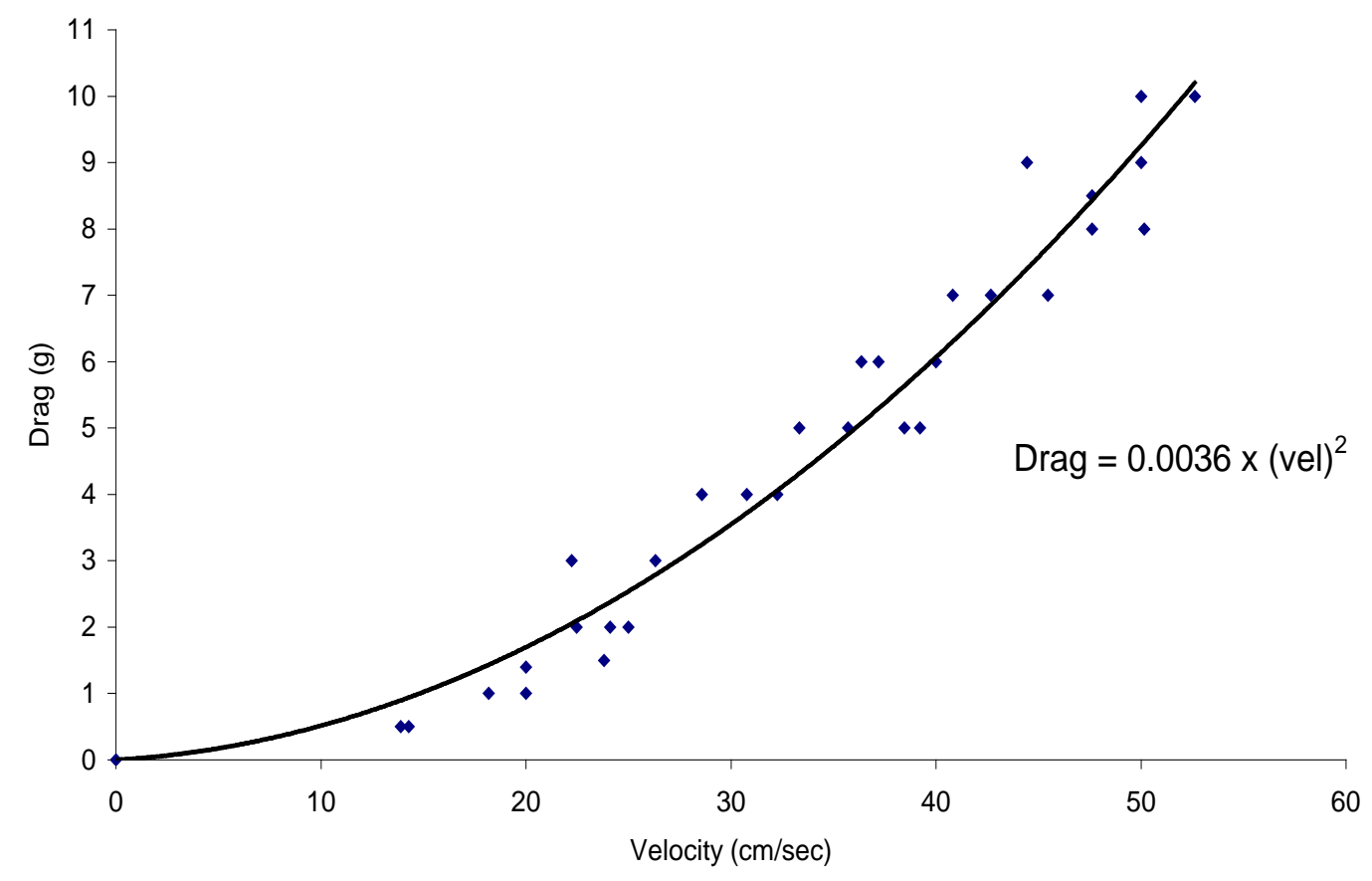

Fig. 8 Craft's drag as a function of its velocity 


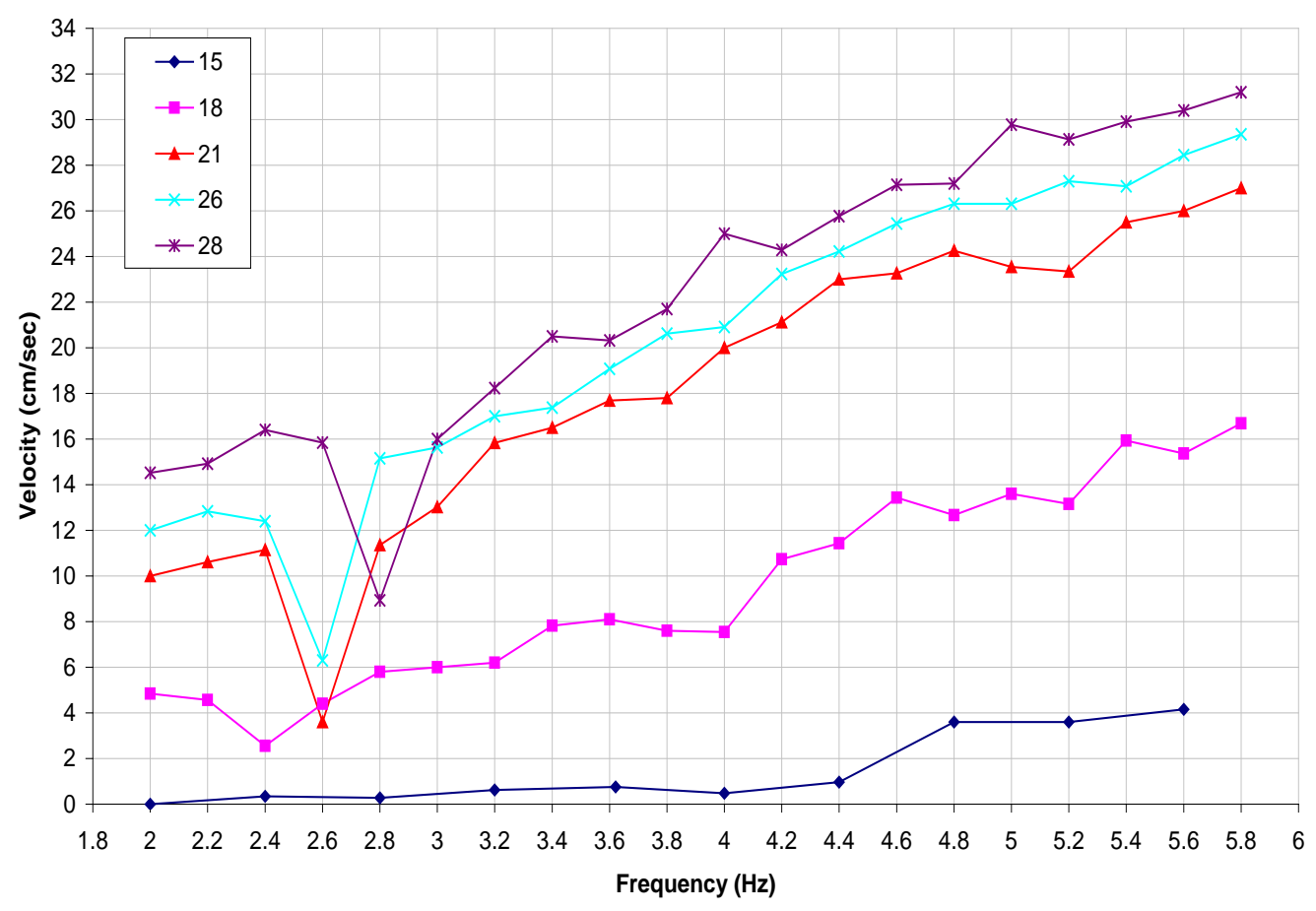

Fig. 9 Steady state craft velocity as a function of frequency and amplitude 


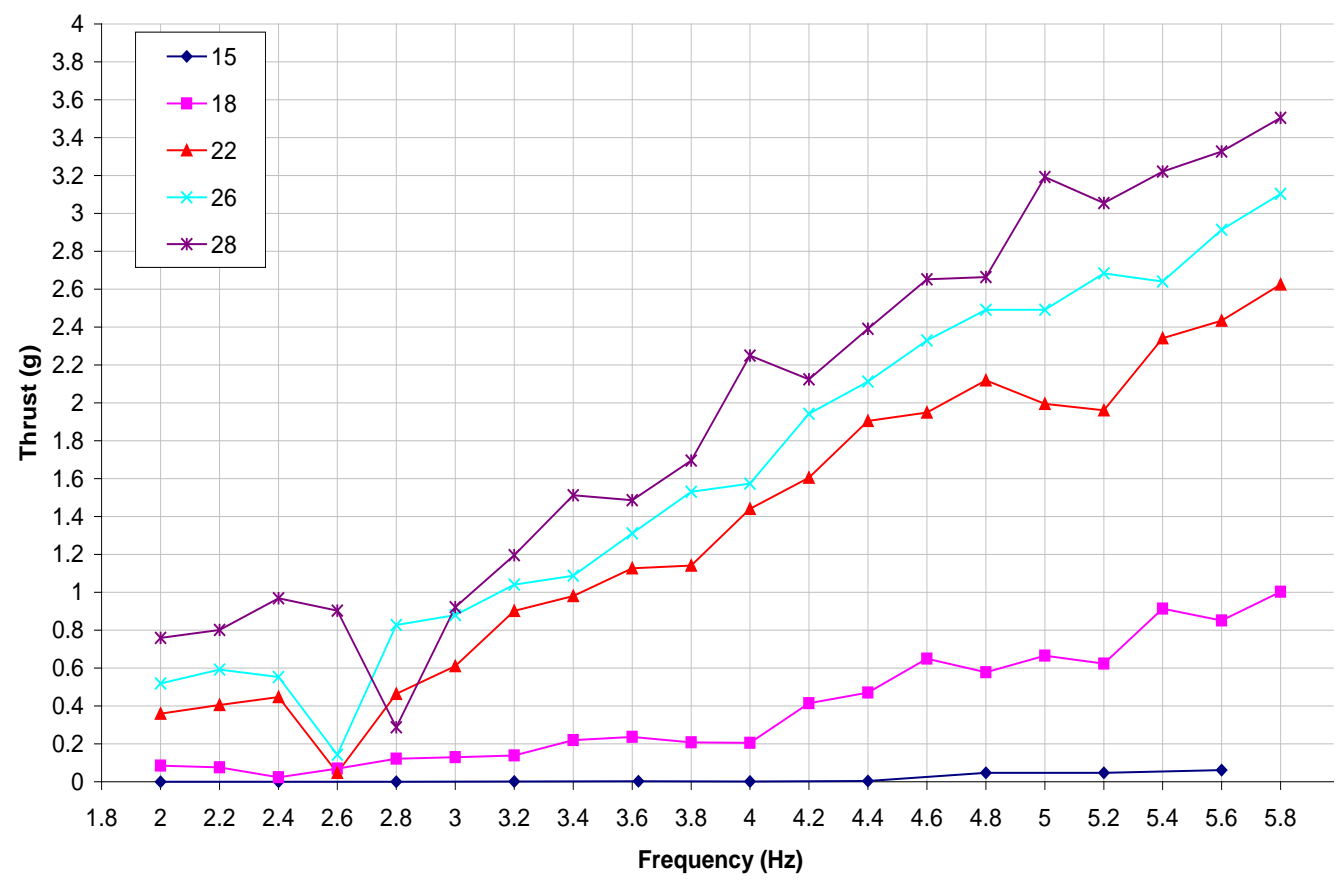

Fig. 10 Variation of self-propulsion thrust with frequency and amplitude 


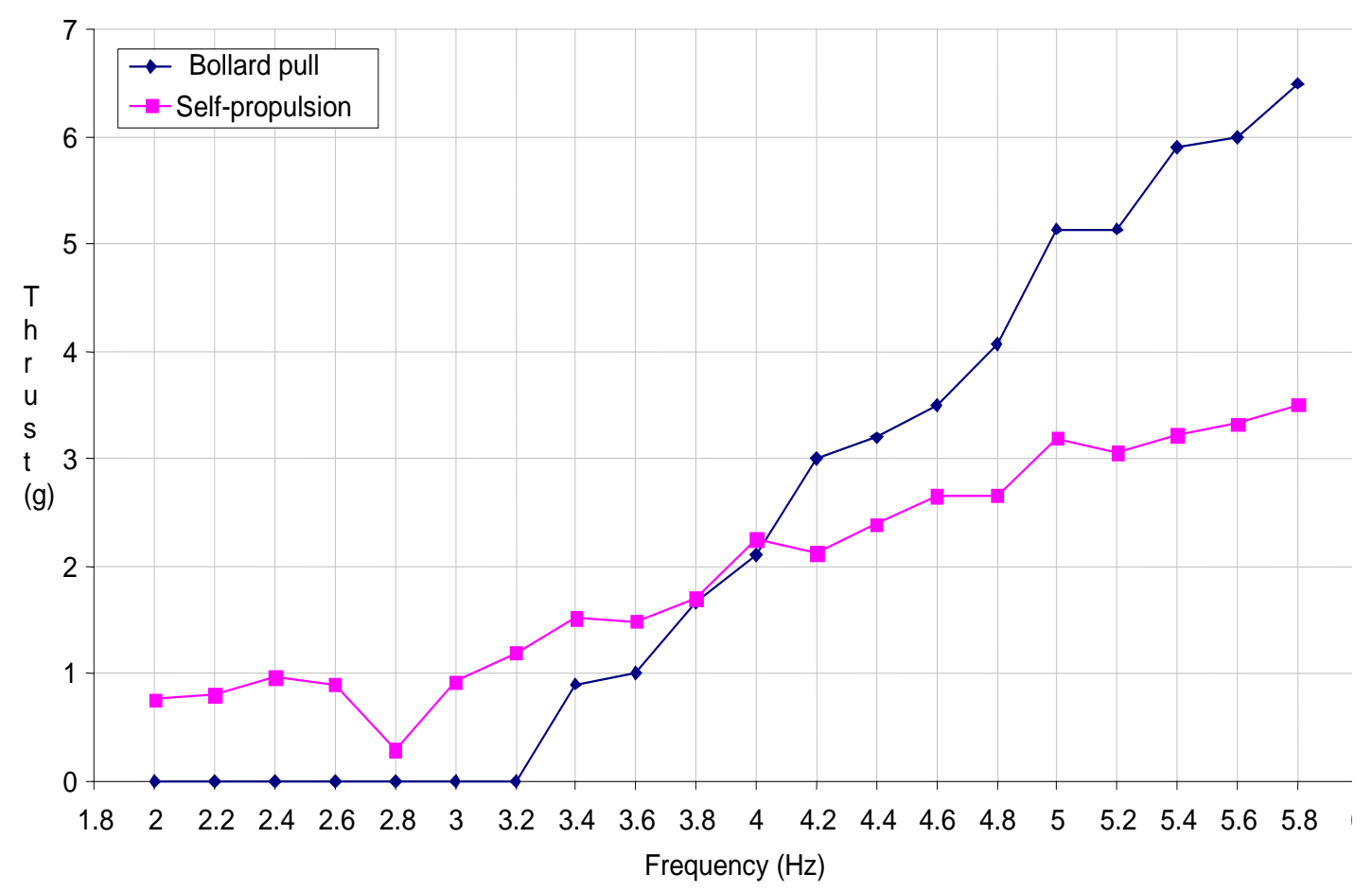

Fig. 11 Bollard pull and self-propulsion thrusts at $28 \mathrm{~mm}$ amplitude 